\title{
Formation of Icosahedral Aluminum-Manganese by Electron, Laser and Ion Beams
}

\author{
J. A. Knapp and D. M. Follstaedt \\ Sandia National Laboratories \\ Albuquerque, NM 87185, USA
}

\begin{abstract}
Last year at the National Bureau of Standards, examination of certain aluminummanganese alloys which had been melted and the rapidly cooled on a spinning copper wheel showed that the atomic arrangement of the resulting solid exhibited long-range order with icosahedral orientational symmetry. This means, for example, that certain directions show five-fold rotational symmetry in electron diffraction patterns. The discovery is surprising because five-fold rotational symmetry is impossible for a crystalline material, and all previously examined solids with long-range order have been found to be crystalline.
\end{abstract}

The problem is illustrated by an analogy in two dimensions: a floor cannot be tiled using a periodic array of pentagons (fivefold symmetry) without either overlapping tiles or leaving gaps. Naturally, the observation of an ordered material exhibiting icosahedral symmetry has generated intense interest. Currently, many workers interpret the experimental observations in terms of a "quasicrystalline" atomic arrangement. Again, the analogy of tiling a floor is helpful: a floor can be covered using two differently shaped tiles in a non-periodic array such that the overall symmetry of the pattern is five-fold. The pattern does not repeat, so no two areas of the floor have exactly the same local arrangement of tiles.

Such a pattern is called a "Penrose tiling," after its inventor. A "quasicrystal" would be a three-dimensional Penrose tiling, where two different groupings of atoms are arranged in a non-periodic array to form the solid. Such a solid would be able to diffract electrons like a crystal, but symmetries such as icosahedral would be allowed. If this explanation is correct, quasicrystals are an entirely new class of ordered materials.

In order to determine the properties of the "icosahedral phase" and to explore other techniques which might lead to its formation, we have examined thin surface layers of aluminum-manganese produced by surface alloying techniques. First, alternating aluminum and manganese layers are deposited onto an aluminum or iron substrate to a total thickness of 50-100 nm. This surface layer is then treated with an electron beam, a laser beam, or a highenergy ion beam to form the icosahedral phase. If a laser or fast electron beam treatment is used, the surface layer is melted and then frozen very quickly (within less than one millionth of a second); this treatment produces a layer of small icosahedral particles, typically $30 \mathrm{~nm}$ in diameter. If a slower electron beam treatment is used, and the substrate is iron, the molten layer cools more slowly and grains of the icosahedral phase up to a micrometer in diameter are formed. Although generally similar to other liquid quenching techniques which have been used to form the icosahedral phase, the laser and electron beam treatments have well-known temperature histories which allow us to place limits on the melting point of the icosohedral phase (between 660 and $960^{\circ} \mathrm{C}$ ) and the time needed for the phase to start forming (less than $20 \mathrm{~ns}$ ).

The other method of reacting the layers involves bombarding the sample with highenergy ions, and is called ion-beam mixing. This is a new way of forming the icosahedral phase which is quite different from melt quenching. A beam of xenon ions accelerated to an energy of $400 \mathrm{keV}$ is used to intermix the layers on an atomic scale by producing localized atomic displacements. The xenon ions pass through the layers and come to rest in the substrate underneath; they do not become part of the alloy. By bombarding the layers for a sufficient time with the material heated to $80-100^{\circ} \mathrm{C}$, the aluminum and manganese are converted into small grains of the icosahedral phase. If the same bombardment is done with the material held at room temperature, the layers are instead converted to an amorphous phase, which has no long-range ordering at all, either crystalline or quasicrystalline. This amorphous (or glassy) layer can then be converted to the icosahedral phase by heating the sample to $300^{\circ} \mathrm{C}$. Further heating to $400^{\circ}$ converts the layer to a crystalline phase.

These studies are providing fundamental information about such things as the melt times and cooling rates needed to form this new phase. These unique materials may prove to have unusual and useful physical properties; if so, the information our work provides will be needed to help develop practical applications of "quasicrystals."

MIRIS

\section{Carbon in Crystalline Silicon}

\author{
Ronald C. Nerwan \\ University of Reading \\ Reading, UK
}

Atoms of a crystal are arranged in a regular array to form a lattice which is analogous to a stack of chess boards one on top of the other, with one atom located on each square. The geometrical arrangement adopted depends on the constituent atoms and is different for iron, copper, or silicon. It has been found that each silicon atom links or bonds to four neighboring silicon atoms to form a pyramidal or tetrahedral structure, which is exactly the same as that for carbon atoms in diamond. Therefore, it is not surprising that during the growth of a silicon crystal some atoms can simply be replaced by carbon atoms present as an inadvertent impurity. Such a crystal con- tains "imperfections." The incorporation of carbon introduces strain into the crystal which arises because carbon atoms are smaller than silicon atoms. As a consequence, only a limited number of impurities can be present, and this number becomes smaller as the temperature of the crystal is reduced. 


\section{Continued from previous page}

The real situation is more complicated because other impurity atoms will be present, particularly oxygen which is bonded or anchored to two silicon neighbors but is located in the spaces or interstices between the silicon atoms. The oxygen strains the crystal in the opposite sense from carbon, and the two types of impurity prefer to occupy adjacent sites as this leads to an overall reduction in the strain. To form such pairs the carbon impurity would have to jump from one lattice site to the next, but this is not easily achieved because the atoms of the crystal are in the way and an exchange process must occur. On the other hand, for atoms in interstitial sites where the bonding is weak, migration through the crystal can proceed much more readily.

When silicon crystals are bombarded with nuclear particles, collisions occur and some atoms are knocked off their lattice sites into the interstices. Carbon impurity atoms are very readily transferred during this process and can then migrate even at room temperature. They are then trapped at other imperfections in the crystal including undisplaced carbon and oxygen atoms to form a whole series of complicated clusters of impurities. It is crucially important to understand the structure and nature of such defects because they can have detrimental effects on the properties of silicon used to make (a) integrated electronic circuits or (b) devices for power circuits.

In this paper, we review the current state of knowledge of these effects in silicon immediately after its growth, and following various processing, including high-energy bombardment and heat treatments at elevated temperatures which can also lead to displacement of carbon atoms from lattice sites. The processes are monitored mainly by measurements of transparency of the crystals with optical equipment operating in the infrared spectral region. This diagnostic method can be understood by comparison with other materials that are transparent to visible light. The presence of defects or impurities in such a material, including glass, removes light at certain wavelengths and the material acquires a characteristic color. The view of silicon in the infrared is similarly "colored" by the defects involving carbon and the color changes produced by processing tell us which types of defect have been produced or destroyed. Colors characteristic of several defects have been identified but considerbly more research effort will be needed before a full understanding can emerge.

\title{
Special-Purpose Computer for Molecular Dynamics
}

\author{
H. R. Carleton \\ State University of New York, \\ Stony Brook, Long Island, NY 11794, U.S.A.
}

Physical systems have traditionally been analyzed by searching for functional relationships between various measurable parts of the system such as the density, temperature, particle forces, etc. These functional relationships are represented by complex mathematical functions which are then analyzed by classical methods for the solution of the integro-differential equations. Analytic solutions thus obtained can then be evaluated on a computer to obtain numerical results for further study. This method is severely limited when applied to many interacting particles because of the huge number of equations which would be required and because the solutions, when obtainable, are the sum of a large number of functions.

With the advent of high-speed digital computers it is feasible to approach the understanding of physical systems by a direct calculation of the behavior of each individual element of the system, from which global parameters can be derived. This is possible because the computer has the unique ability to keep track of a very large amount of information at the same time and also because it is much faster at simple calculations than the scientist. A direct calculation of the behavior of each atom or particle in a material based on its motion over a very short period of time can be easily written as a large number of simple equations. This approach to materials properties has been dubbed, "molecular dynamics" and is one example of the general approach to solving problems by direct simulation of the behavior of each element of a system.

Such an approach is not a panacea, however, when the amount of brute-force calculation required to simulate even simple systems is considered. For example, it is easy to show that a computer which is capable of computing the interaction of any pair of particles in a system as small as a grain of table salt in one-millionth of a second (a microsecond) would require $10^{44}$ years of calculation to simulate the salt crystal for one second of real time, since each atom in the grain interacts with all other atoms.

Obviously, compromises must be made to achieve a reasonable computation time. Study has shown that a good model of a crystal can be made with about 1000 atoms. In addition, it is sufficient to study the crystal over shorter periods, say 0.001 second. With these new numbers a reasonable computation can be carried out in about one year if we can afford the fulltime cost of a super-minicomputer for that period.

Since a computer must be virtually dedicated to a single problem for simulations of this size (and hopefully much larger) it is logical to build a special-purpose computer which is better at simulation than a generalpurpose computer. Such an approach is made feasible with the availability of inexpensive, monolithic computing elements which each have a computation speed comparable to the arithmetic component of a superminicomputer.

The calculations that must be carried out in molecular dynamics between each pair of particles is relatively simple, so it is practical to provide a large number of these computing elements to achieve a further increase in computing speed proportional to the number of arithmetic processor elements used. In this way, computations for different particles are carried out simultaneously by different computer processors. Since the computations carried out on different particles are virtually the same in a molecular dynamics calculation, such a problem is ideal for the application of multiple processors.

The use of multiple processors in this way is called parallel processing, and is being widely applied in the computer industry today. There are many problems in achieving a practical parallel processor system related to the transfer of information between processing elements. However, there is a great cost advantage in building a parallel processor system when the class of problem that must be computed

Continued 